\title{
Load Flow Analysis with Analytic Derivatives for Electric Aircraft Design Optimization
}

\author{
Eric S. Hendricks, ${ }^{*}$ Jeffryes W. Chapman, ${ }^{\dagger}$ and Eliot D. Aretskin-Hariton ${ }^{\ddagger}$ \\ NASA Glenn Research Center, Cleveland, Ohio, 44135
}

\begin{abstract}
Many of the aircraft concepts of the future are exploring the use of hybrid-, turbo- or allelectric propulsion systems to improve performance and decrease environmental impacts. These aircraft concepts range from small rotorcraft for urban air mobility to conventional commercial transports to large blended wing body designs. Developing the conceptual design for these vehicles presents a challenge, however, as traditional aircraft design tools often were not developed to handle these unique propulsion system architectures. Previous studies on these vehicles have therefore relied on relatively simple models of the electrical transmission and distribution system. This paper presents the development of a hybrid AC-DC load flow (or power flow) analysis capability to enhance the conceptual design of these concept vehicles. Specifically, the desire was to create a load flow analysis capability within the OpenMDAO framework that is also being used to develop a set of compatible tools for rapid optimization of conceptual designs. This load flow analysis capability is unique in its flexible object-oriented structure and implementation of analytic derivatives to facilitate the use of solvers and gradient based optimization in the design process. The developed hybrid load flow analysis capability is first verified against a published 13-bus example then used to model the electrical distribution system for a turbo-electric tiltwing aircraft.
\end{abstract}

\section{Nomenclature}

$M \quad$ Converter modulation index

$P \quad$ Real power

$P F \quad$ Power factor

$Q \quad$ Reactive power

$R \quad$ Resistance or rectifier

$S \quad$ Apparent power

$V \quad$ Voltage

$X \quad$ Reactance

$Y \quad$ Admittance

$Z \quad$ Impedance

$\theta \quad$ Phase angle

$\eta \quad$ Converter efficiency

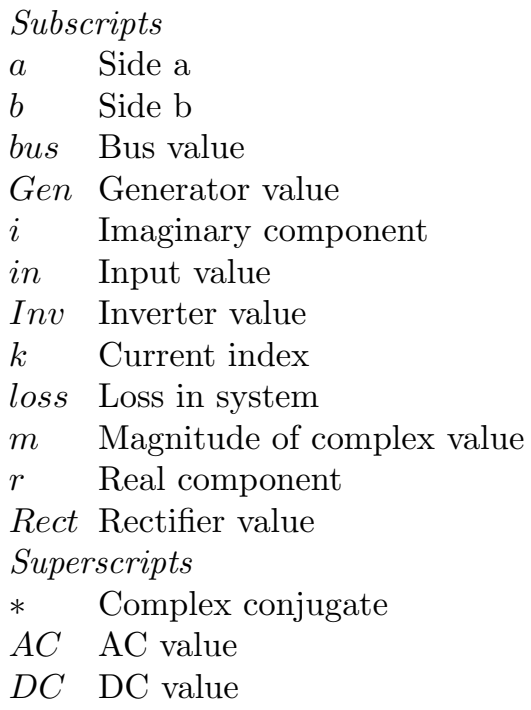

*Aerospace Engineer, Propulsion Systems Analysis Branch.

$\dagger$ Aerospace Engineer, Propulsion Systems Analysis Branch.

$¥$ Aerospace Engineer, Intelligent Control and Autonomy Branch. 


\section{Introduction}

IN 2017, NASA's Aeronautics Research Mission Directorate released its Strategic Implementation Plan 1 which outlines a vision for aeronautical research. ${ }^{1}$ This document describes six strategic research thrusts which were identified by evaluating overarching drivers that are expected to shape the future aviation industry. These research thrusts include Thrust 3: Ultra-Efficient Commercial Vehicles and Thrust 4: Transition to Alternative Propulsion and Energy. To support these research objectives, "NASA is investing in Electrified Aircraft Propulsion (EAP) research as part of the portfolio to improve the fuel efficiency, emissions, and noise levels in commercial transport aircraft." ${ }^{2}$ The focus on a EAP covers a range of different propulsion system architectures which have been proposed for a variety of future aircraft conceptual designs. These conceptual designs include NASA's X- $57,{ }^{3}$ STARC-ABL $,{ }^{4} \mathrm{~N}-3 \mathrm{X},{ }^{5}$ and several urban air mobility (UAM) designs shown in Figure $1 .{ }^{6}$ These NASA concepts are supplemented by several proposed by industry including the Boeing SUGAR Volt ${ }^{7}$ and ESAero ECO-150. ${ }^{8}$
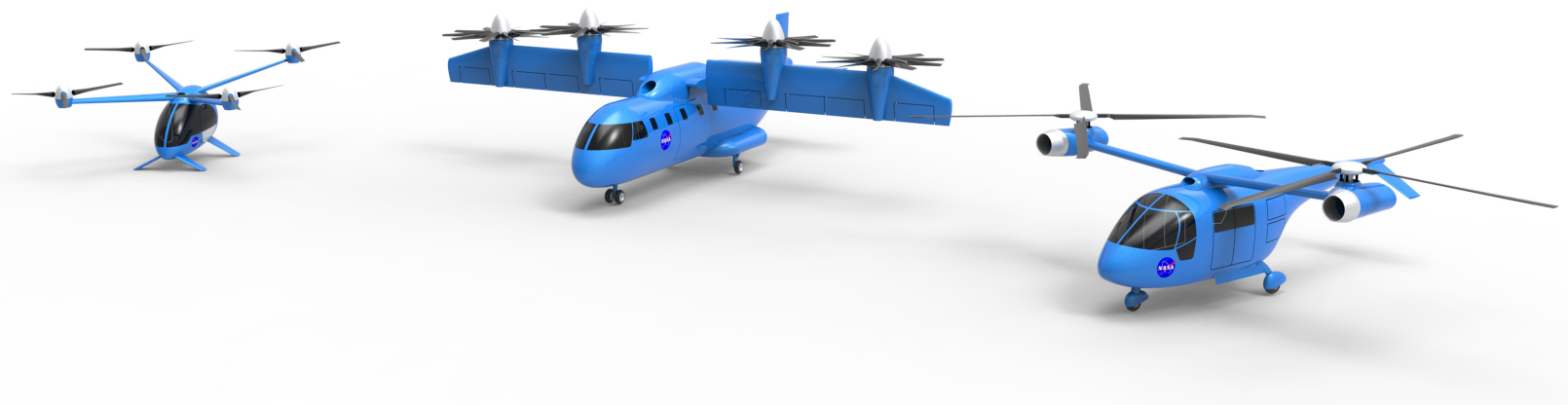

Figure 1. NASA Urban Air Mobility Concept Vehicles.

Developing the conceptual designs for these vehicle configurations presents a number of challenges, particularly in relation to the electrified propulsion systems. The primary challenge is that many of the traditional conceptual level design tools were not developed evaluate these unique configurations. Therefore, many of the preliminary systems analysis studies have utilized relatively simple models of the electrical power distribution system. For example, Welstead and Felder ${ }^{4}$ analyzed the STARC-ABL concept by specifying a set of efficiencies for the components expected to be require. These efficiencies were based on assumptions about future technology levels and provided a simple means to determine the losses through each component. A similar approach was taken in an analysis of the X-57 by Falck et al. ${ }^{9}$ While this approach provides a rapid systems analysis capability, it also lacks important details that may be critical to evaluating the electrical power system.

One approach for enhancing the propulsion and power system analysis capability for these concepts can be taken from the traditional load flow (or power flow) method. ${ }^{10}$ This method is traditionally used by power engineers to model interconnected electrical systems such as the terrestrial AC power grid. However, it has also been extended to analyze DC and hybrid AC-DC systems ${ }^{11}$ as well as isolated micro-grids. One of the benefits of the load flow method for EAP concepts is that it predicts voltages and currents throughout an electrical network given the states of distributed electric generators and loads. The approach therefore naturally lends itself to analyzing electric aircraft propulsion systems that may include a number of generators and motors. Additionally, the approach is commonly used to determine the optimal power flow and optimal operations of groups of generating units or to consider short circuit fault scenarios. These applications are critical for the development of efficient and robust electric aircraft propulsion systems. Overall, the load flow approach is a well established technique but has not been extensively applied in the conceptual design of aircraft propulsion systems. Furthermore, the additional information provided by a load flow analysis is likely to be beneficial for conceptual aircraft design studies.

This paper describes the development of a load flow analysis capability to support the conceptual design of electric aircraft propulsion systems. While other load flow tools exist, one specifically for EAP control studies, ${ }^{12}$ none provide the connectivity and design capability desired for these studies. Therefore, Section II describes the development of an object-oriented hybrid AC-DC load flow analysis code that provides analytic 
derivatives to support design optimization studies. Following this development, the load flow code is verified by comparing results to a published thirteen bus hybrid AC-DC system. The load flow analysis code is then used in Section IV to examine the electric distribution system design of a turbo-electric tiltwing aircraft for urban air mobility. Lastly, Section V provides conclusions and future work from this study.

\section{Load Flow Analysis Code Development}

Load flow analysis provides a relatively simple approach for analyzing interconnected AC electrical distribution systems. An example of a simple 3-bus load flow analysis problem is shown in Figure 2. In this example, a single generator $(\mathrm{G})$ with a prescribed voltage magnitude $(\mathrm{V})$ and phase angle $(\theta)$ is attached to bus 1 (represented by a horizontal line). Two loads $\left(\mathrm{L}_{2}\right.$ and $\mathrm{L}_{3}$ ) with specified real $(\mathrm{P})$ and reactive $(\mathrm{Q})$ powers are also part of the system and are attached to bus 2 and bus 3 respectively. Between each of the buses is a transmission line, which although drawn as a single line, actually represents a three phase power system. The transmission line characteristics are defined by the resistance (R) and reactance (X) which collectively are referred to as the impedance.

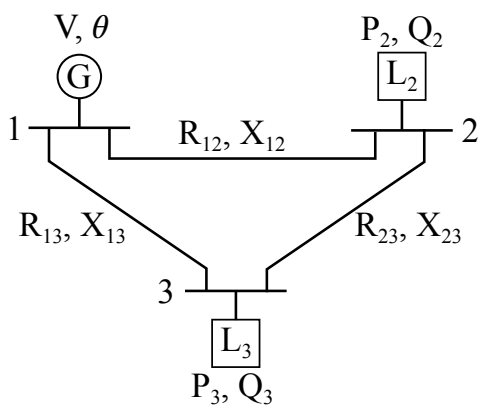

Figure 2. Three bus load flow model.

Given this input information for the generator, loads, and lines, the objective of load flow analysis is to determine the voltages, currents, and powers throughout the system. The traditional approach for determining these values is to first form an admittance matrix based on the line impedance values. This matrix is then multiplied by a vector of initial guess for the bus voltages to compute the currents entering and leaving each of the buses. Numerical methods such as a Newton-Raphson or Gauss-Seidel are then applied to determine the actual voltage for each of the buses needed to make the sum of the currents entering and leaving the bus equal zero. After the bus voltages are determined, the currents and powers throughout the system are then computed.

For this work, the goal was to develop a flexible load flow analysis capability that could be efficiently integrated into the conceptual design and optimization process for electric aircraft. While this traditional approach works well for conventional load flow studies, the basic process and traditional tools do not support gradient based optimization of these aircraft power systems which may contain both AC and DC components. Therefore, this research effort focused on creating a new hybrid AC-DC load flow analysis capability capable of modeling these systems with additional focus on supporting gradient based optimization through the implementation of analytic derivatives.

To develop this new modeling capability, the OpenMDAO framework ${ }^{13,14}$ was selected as the software basis due to several valuable features. First, OpenMDAO provided a flexible tool and modeling structure as it is an open source, object-oriented framework written in the python programming language. Of specific interest, the framework provides two base classes which provide the needed analysis and derivative calculation methods required to completely define the load flow systems. The first base class is an explicit component which computes a set of output values $(\bar{y})$ based on a set of inputs $(\bar{x})$ following the generic expression in Equation 1.

$$
\bar{y}=f(\bar{x})
$$

The second base class of interest in OpenMDAO is an implicit component. This base class defines a set of 
nonlinear residual equations of the form given in Equation 2.

$$
R(\bar{x}, \bar{y})=0
$$

Here, the outputs $(\bar{y})$, also referred to as state variables, are determined by finding the values that make the residual equations true within a defined tolerance. The implicit component base class therefore provides an avenue for easily implementing a set of nonlinear equations which can be numerically converged through the use of a solver. Second, the OpenMDAO framework contains a number of linear and nonlinear solvers (such as Newton-Raphson) as well as gradient-based optimization algorithms which are required to solve and design the electric propulsion systems. These solvers and optimizers typically require the accurate and rapid calculation of gradient information to efficiently find solutions. OpenMDAO provided unique capabilities for analytically computing the partial derivatives of individual components in order to determine the overall total derivatives needed to facilitate the use of gradient based solvers and optimizers. ${ }^{15,16}$ Furthermore, the OpenMDAO framework is being used to develop and integrate a set of compatible tools for other disciplinary analyses needed for rapid optimization of conceptual electric aircraft designs.

Given these valuable traits of OpenMDAO, the calculations needed for a hybrid AC-DC load flow capability were implemented within the framework and will be described in the subsequent sections. While load flow analysis is typically only applied the AC systems, a hybrid AC-DC load flow method ${ }^{11}$ was identified that enabled creation a system to capture both types of electrical systems. From this publication, a set of 10 objects which would be needed to model almost any electrical system were identified. These objects include AC and DC versions of the bus, line, generator, and load. In addition, components modeling an inverter and a rectifier are required to convert between the two different voltage sources. The sections below describe the $\mathrm{AC}$ versions of the bus, line, generator and load components as well as the converter which were developed in this research. The DC components are not described but are identical to their AC equivalents with the exception of the imaginary terms and phase angles in the equations. This separation of AC and DC components was made to maintain clarity in the use of these components and to simplify the computation of analytic derivatives in each of the developed components. In addition to the equations presented in each of the sections below, analytic expressions for partial derivatives of each output with respect to the each input were determined by symbolically differentiation and included in the code. These partial derivatives are key to enabling the rapid solution of the load flow problem as well as for multidisciplinary optimization around a load flow model.

\section{Line}

The line component is written as an explicit component in OpenMDAO such that it directly computes outputs based on inputs. The inputs to the line component are the resistance, reactance and the voltages (real and imaginary parts) of the buses at each end of the line. Given these inputs, the admittance is first computed using Equation 3. Next, the current at the two ends of the line (labeled $a$ and $b$ ) are given by Equation 4. Lastly, the line power at each end is computed using Equation 5 and the power loss through the line is computed following Equation 6 .

$$
\begin{aligned}
& Y=\frac{1}{R+X i} \\
& I_{a}=Y\left(V_{a}-V_{b}\right) \quad I_{b}=Y\left(V_{b}-V_{a}\right) \\
& S_{a}=V_{a} I_{a}^{*} \quad S_{b}=V_{b} I_{b}^{*} \\
& S_{\text {loss }}=S_{a}+S_{b}
\end{aligned}
$$

\section{Load}

The load component is also formulated within OpenMDAO as an explicit component. This component has inputs which specify the real and reactive power demanded by the load. In addition, the component also 
requires as input the voltage of the connected bus. With this limited set of inputs, the component computes the current required by the load using Equation 7 .

$$
I_{\text {load }}=\left(S / V_{\text {bus }}\right)^{*}
$$

\section{Bus}

While the line and load can be implemented as explicit components, the bus component is written in OpenMDAO as an implicit component. This means that the component's outputs are a set of state variables with associated residual equations which must be determined by a numerical solver. For the bus, the output state variables are the real and imaginary components of the voltage on the bus (or the voltage magnitude and phase angle). The inputs to the bus are the currents from each of the lines, loads or generators attached to the bus. The residual equations implemented in the bus component require that the sum of the currents (both real and imaginary parts) entering the bus must equal zero as defined in Equations 8 and 9. While the voltage state variables are directly included in these equations, they are indirectly included as the voltage on the bus affects the currents computed by the lines, loads and generators.

$$
\begin{aligned}
& \Sigma I_{r, k}=0 \\
& \Sigma I_{i, k}=0
\end{aligned}
$$

\section{Generator}

The generator, also an implicit component, is the most complex of the elements as it has two different operating modes depending on the type of generator being considered. The first mode is for a swing or slack generator. In this mode, the component has inputs for the target voltage magnitude and phase angle of the attached bus. In addition, the component takes in the present value of the voltage on the connected bus. The output state variables for the generator are the real and imaginary parts of the current sent by the generator to the bus. Using this inputs and state variables, the residual equations for the generator component are given in Equations 10 and 11. These two equations state that the voltage magnitude and phase angle specified as target values by the user must be matched by the actual voltage and phase angle from the bus. Again, the output state variables for the current are not directly included in these residual equations. However, the current state variables indirectly influence the residual equations as they change the currents entering the bus and therefore alter the voltage and phase angle required to satisfy the bus equations. As a result, using the real and imaginary parts of the current as output state variables still results in a valid system of nonlinear equations.

$$
\begin{gathered}
V_{m, \text { in }}-V_{m, \text { bus }}=0 \\
\theta_{\text {in }}-\theta_{\text {bus }}=0
\end{gathered}
$$

The second mode of operation for generator components is for a power-voltage controlled generator. For this mode, the real power and voltage magnitude supplied by the generator to its associated bus are specified as inputs. The first residual equation for this component is the same as Equation 10, which ensures the voltage magnitude produced by the generator matches that specified as input. The second residual equation is different as it ensures the real power produced by the generator matches the input value for the bus as given in Equation 12. Similar to the slack generator, the power-voltage generator has state variables for the real and imaginary parts of the current sent by the generator to the bus.

$$
P_{\text {in }}-P_{b u s}=0
$$

\section{Inverter}

In addition to the basic components needed to model $\mathrm{AC}$ and $\mathrm{DC}$ systems, two components were created to convert between AC and DC portions of a hybrid power system. The first of these components was the inverter for DC to AC conversion. Modeling the inverter component was completed in a slightly different 
fashion than the previously described components. Rather than create a single custom component, the inverter was created as an OpenMDAO group of three subcomponents: a DC load, an AC generator (in slack mode), and a custom object computing the relationships between the AC and DC sides. This approach was selected as the inverter acts as a load on the DC portion of the system and as a generator on the AC portion. As a result, the existing code for DC load and AC generator could be reused with a simple implicit component created to ensure the proper relationships between the AC and DC sides are maintained. These AC-DC implicit relationships were adapted from the simple model described by Ahmed, Eltantawy and Salama. ${ }^{11}$ The first relationship, given in Equation 13, states that the voltage on AC is equal to the DC voltage multiplied by a modulation index $(M)$. Equation 14 states that the AC power leaving the inverter is equal to the inverse of the DC power entering times the inverter efficiency (note that power entering and leaving the inverter are of opposite sign). The inputs to these equations are $V^{D C}, P^{A C}, M$, and $\eta$ while the output state variables are $V_{m}^{A C}$ and $P^{D C}$.

$$
\begin{gathered}
V_{m}^{A C}-M V^{D C}=0 \\
P^{A C}+P^{D C} \eta=0
\end{gathered}
$$

In addition to these two equations, one additional implicit relationship needed to be defined to fully determine the $\mathrm{AC}$ current exiting the inverter. For this relationship, two different modes of inverter operation were defined. The first mode mimics the slack generator and allows the user to define voltage phase angle of the attached AC bus following Equation 11. This mode of operation allows an inverter to be the sole source of $\mathrm{AC}$ power in an isolated portion of the $\mathrm{AC}$ electrical system (i.e. that portion of the $\mathrm{AC}$ system does not have a slack generator). The second operating mode allows the user to specify the power factor (PF) for the power supplied by the inverter. Specifying the power factor determines the reactive power using Equation 15. For both of these modes of operation, $\theta_{i n}$ is used as the output state variable.

$$
\frac{P^{A C}}{\sqrt{\left(P^{A C}\right)^{2}+\left(Q^{A C}\right)^{2}}}-P F=0
$$

\section{Rectifier}

The second component created for linking the AC and DC portions of a hybrid electrical system was the rectifier. This component followed a similar modeling approach as the inverter with the model being a group composed of three subcomponents: an AC load, a DC generator and a custom implicit object to define the relationships across the rectifier. The implicit relationships for the rectifier are given in Equations 16, 17 and 18. The first two equations again define the voltage and power relationships across the converter based on an input modulation index and efficiency. The last equation computes the $\mathrm{AC}$ reactive power based on an input power factor. For this implicit component, the inputs are $V_{m}^{A C}, P^{D C}, M$, and $\eta$ while the output state variables are $V^{D C}, P^{A C}$, and $Q^{A C}$.

$$
\begin{gathered}
V^{D C}-M V_{m}^{A C}=0 \\
P^{D C} \eta+P^{A C}=0 \\
P^{A C} \sqrt{\frac{1}{P F^{2}}-1}-Q^{A C}=0
\end{gathered}
$$

\section{Hybrid AC-DC Load Flow Model Verification}

Section II described the development of 10 individual components within the OpenMDAO framework that can be combined to model a wide variety of hybrid AC-DC electrical systems. While the equations described in the previous section are relatively simple, the next step in the development process was to verify the load flow calculations were implemented correctly. The verification effort described in this section was completed by examining two different aspects of the load flow calculations. The first verification step examined the functional equations used to create the individual components which were described in the previous section. 
This examination was completed by modeling a reference system and ensured the developed components could reproduce a known load flow solution. The second verification step evaluated the analytical expressions included in the load flow components to compute derivatives. These analytical derivative calculations were included in the developed tool to support of the Newton-Raphson solver and gradient based optimization algorithms.

For the first verification step, the load flow components were combined to create a reference hybrid 13-bus system shown in Figure $3 .{ }^{11}$ This system was selected as it includes instances of all 10 objects described in the previous section and was published with voltage and power results that would enable verification. While the results for the reference system are well documented, it should be noted that the impedance values specified in the publication for the system are incorrect. These values were found to be inconsistent with the published voltage and power results and were therefore not used in this study. Instead, the impedance values computed from the published voltage and power given in Table 1 were used in this study.

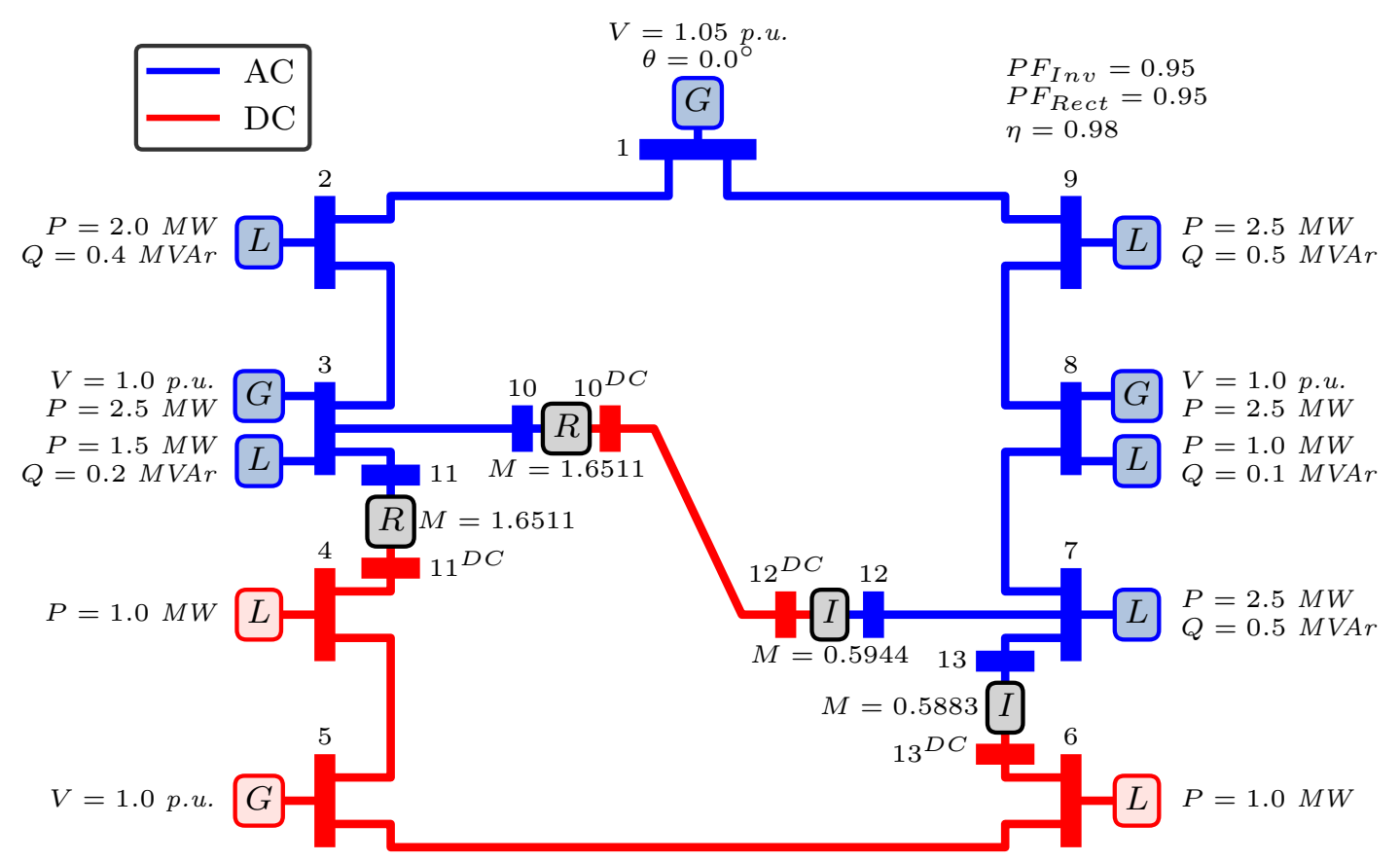

Figure 3. Hybrid AC-DC Load Flow Example. ${ }^{11}$

With these updated line impedances, the 13-bus system was recreated using the components developed in OpenMDAO and its included Newton solver. The bus voltages produced by this model are given in Table 2 along with the voltages given in the reference publication. Overall, the voltage magnitudes for all the buses show excellent agreement with the published data as the maximum error is less than $0.15 \%$. The voltage phase angles also show good agreement but have larger errors in the range of 1.5 to $4.5 \%$. While these phase angle errors are larger than desirable, they were deemed acceptable for verification purposes given the uncertainty in the line impedances described above. In addition to examining the bus voltages, the line powers were also compared to the published data with the results given in Table 3 . The real and reactive power values given in the table again show good agreement with the published values as the relative error is generally less than $5 \%$ for both quantities. These larger errors were again less than desirable but deemed acceptable for initial verification considering the line impedance uncertainty.

The second step in the verification process for the developed load flow analysis capability was to examine the analytically defined derivatives for the reference system. These derivatives are used to determine the search direction for the Newton as well as identify the direction of improvement for gradient based optimizers. Verifying the analytical derivatives computed by the load flow components was completed in two phases. In the first phase, the partial derivatives for each of the individual components described in Section II were 
Table 1. Impedances of the 13-Bus System

\begin{tabular}{cccc}
\hline From Bus & To Bus & Resistance $(\Omega)$ & Reactance $(\Omega)$ \\
\hline 1 & 2 & 0.218734378 & 0.360978021 \\
1 & 9 & 0.224454412 & 0.36093195 \\
2 & 3 & 0.892493161 & 1.542190824 \\
3 & 10 & 0.056583399 & 0.749529076 \\
3 & 11 & 0.040822812 & 0.750680512 \\
4 & 5 & 0.25349905 & - \\
4 & 11 & 0.509605338 & - \\
5 & 6 & 0.209734086 & - \\
6 & 13 & 0.493075443 & - \\
7 & 8 & 0.438930098 & 0.726902905 \\
7 & 12 & 0.03336043 & 0.741618427 \\
7 & 13 & 0.021166743 & 0.766806199 \\
8 & 9 & 0.397894209 & 0.758227135 \\
10 & 12 & 0.892317972 & - \\
\hline
\end{tabular}

Table 2. Bus Voltage Verification with the 13-Bus System

\begin{tabular}{ccccc}
\hline & \multicolumn{2}{c}{ Results } & \multicolumn{2}{c}{ Published Results ${ }^{11}$} \\
Bus & $\mathrm{V}$ (p.u.) & $\theta$ (deg) & $\mathrm{V}$ (p.u.) & $\theta$ (deg) \\
\hline 1 & 1.0500 & 0.0000 & 1.0500 & 0.0000 \\
2 & 1.0130 & -2.0769 & 1.0130 & -2.0627 \\
3 & 1.0000 & -2.5419 & 1.0000 & -2.4637 \\
4 & 0.9970 & - & 0.9970 & - \\
5 & 1.0000 & - & 1.0000 & - \\
6 & 0.9939 & - & 0.9940 & - \\
7 & 0.9471 & -6.4580 & 0.9460 & -6.6463 \\
8 & 1.0000 & -2.8475 & 1.0000 & -2.9794 \\
9 & 1.0041 & -2.5937 & 1.0040 & -2.6356 \\
10 & 0.9878 & -4.1694 & 0.9880 & -4.0680 \\
11 & 0.9920 & -3.6881 & 0.9920 & -3.6096 \\
12 & 0.9572 & -4.7770 & 0.9560 & -4.9847 \\
13 & 0.9523 & -5.5580 & 0.9510 & -5.7869 \\
\hline
\end{tabular}

compared to those computed using finite difference approximation. For the explicit components, this process computed the partial derivative of each output with respect to all of the input variables. For the implicit components, the partial derivatives of the residual values were computed with respect to both the input and output state variables. These comparisons for the explicit and implicit components found that the analytical derivative expressions computed derivative values that were closely matched by finite difference. This result therefore provided a strong indication that the analytic partial derivatives for each component were defined correctly.

The second phase of the derivative verification examined the computation of total derivatives for the hybrid 13-bus system. These total derivatives span multiple components and determined by combining the appropriate partial derivatives defined in the individual components. Verification of these total derivatives again was completed by comparing derivative values to those determined by finite difference approximation. For the finite difference approximation, a relative step size of $10^{-6}$ was used with the solver relative tolerance at $10^{-10}$. A portion of the results from the total derivative verification are shown in Figure 4. This figure shows the absolute error of the derivatives computed analytically with those computed for finite difference for select bus voltage magnitudes with respect to changes in the real and reactive power inputs for each of the loads. For example, the upper left square in the figure shows that the absolute error of $\frac{\mathrm{d} V_{2}}{\mathrm{~d} P_{2}}$ computed by the two methods was $2.8 \mathrm{E}-4$. Overall, the figure shows the analytic and finite difference computed derivatives closely match as the maximum absolute error is on the order of $10^{-2}$ with the relative errors typically being much smaller. The results of the total derivative verification therefore found that the analytic derivatives were properly implemented thereby providing accurate derivative information for use by both solvers and optimizers. 
Table 3. Line Power Verification with the 13-Bus System

\begin{tabular}{cccccc}
\hline & & \multicolumn{2}{c}{ Results } & \multicolumn{2}{c}{ Published Results ${ }^{11}$} \\
From Bus & To Bus & $\mathrm{P}(\mathrm{MW})$ & $\mathrm{Q}(\mathrm{MW})$ & $\mathrm{P}(\mathrm{MW})$ & $\mathrm{Q}(\mathrm{MW})$ \\
\hline 1 & 2 & 2.1921 & 0.5680 & 2.1830 & 0.5733 \\
1 & 9 & 2.7098 & 0.6791 & 2.7380 & 0.6666 \\
2 & 1 & -2.1333 & -0.4709 & -2.1238 & -0.4764 \\
2 & 3 & 0.1333 & 0.0709 & 0.1238 & 0.0764 \\
3 & 2 & -0.1322 & -0.0690 & -0.1227 & -0.0746 \\
3 & 10 & 0.6659 & 0.2400 & 0.6564 & 0.2365 \\
3 & 11 & 0.4663 & 0.1637 & 0.4663 & 0.1637 \\
4 & 5 & -0.5459 & - & -0.5457 & - \\
4 & $11^{\mathrm{DC}}$ & -0.4541 & - & -0.4543 & - \\
5 & 4 & 0.5475 & - & 0.5471 & - \\
5 & 6 & 1.3385 & - & 1.3230 & - \\
6 & 5 & -1.3304 & - & -1.3147 & - \\
6 & $13^{\mathrm{DC}}$ & 0.3304 & - & 0.3147 & - \\
7 & 8 & -1.5486 & -0.2131 & -1.5729 & -0.2201 \\
7 & 12 & -0.6289 & -0.1865 & -0.6200 & -0.1838 \\
7 & 13 & -0.3225 & -0.1004 & -0.3071 & -0.0960 \\
8 & 7 & 1.6177 & 0.3276 & 1.6452 & 0.3385 \\
8 & 9 & -0.1177 & -0.0308 & -0.1452 & -0.0147 \\
9 & 1 & -2.6180 & -0.5315 & -2.6457 & -0.5156 \\
9 & 8 & 0.1180 & 0.0315 & 0.1457 & 0.0156 \\
10 & 3 & -0.6642 & -0.2183 & -0.6550 & -0.2153 \\
$10^{\mathrm{DC}}$ & $12^{\mathrm{DC}}$ & 0.6509 & - & 0.6419 & - \\
11 & 3 & -0.4657 & -0.1531 & -0.4656 & -0.1530 \\
$11^{\mathrm{DC}}$ & 4 & 0.4564 & - & 0.4563 & - \\
12 & 7 & 0.6299 & 0.2070 & 0.6213 & 0.2042 \\
$12^{\mathrm{DC}}$ & $10^{\mathrm{DC}}$ & -0.6427 & - & -0.6340 & - \\
$13^{\mathrm{DC}}$ & 6 & -0.3292 & - & -0.3137 & - \\
13 & 7 & 0.3226 & 0.1060 & 0.3074 & 0.1011 \\
\hline & & & & &
\end{tabular}

Beyond verifying the output values and analytic derivatives, the evaluation of this reference system was also used to examine the performance of the developed analysis code. For this verification, the initial voltage guesses for the numerical iteration were set at 1 p.u. with a 0 degree phase angle on all buses. Initial guesses for the real power passing through the inverter and rectifier components were also set at \pm 0.6 MW. With these initial guesses, the implemented Newton-Raphson solver converged the system in 4 iterations to a relative tolerance of $10^{-10}$ in less than 0.2 seconds on standard laptop computer. These results show that the hybrid 13-bus reference system can be converged tightly in a minimal number of Newton-Rapshon iterations with low wall time. In summary, this verification study demonstrates that the developed AC, DC and converter components are correct, provide accurate analytic derivatives, and are capable of efficiently modeling relatively complex electrical distribution systems in the support of future multidisciplinary optimizations of electric aircraft conceptual designs.

\section{Load Flow Analysis of a Notional Turbo-Electric Tiltwing Electric Distribution System}

With a verified hybrid AC-DC load flow analysis capability, the next step in this research effort was to apply this tool to the analysis of a UAM tiltwing aircraft pictured in the center of Figure 1. This conceptual vehicle design anticipates that the vehicle will be able to transport 15 passengers over a range of 400 nautical miles making multiple stops along the way. ${ }^{6}$ Given the large size of this vehicle, the initial conceptual design determined that it would likely need to be power by a turbo-electric system. In this system, a turboshaft engine is used to generate electrical power that is then distributed to electrical motors driving the four propellers. The successful design of the electrical distribution system is therefore critical to the overall viability of this concept. While the initial conceptual design determined that the turbo-electric system would 


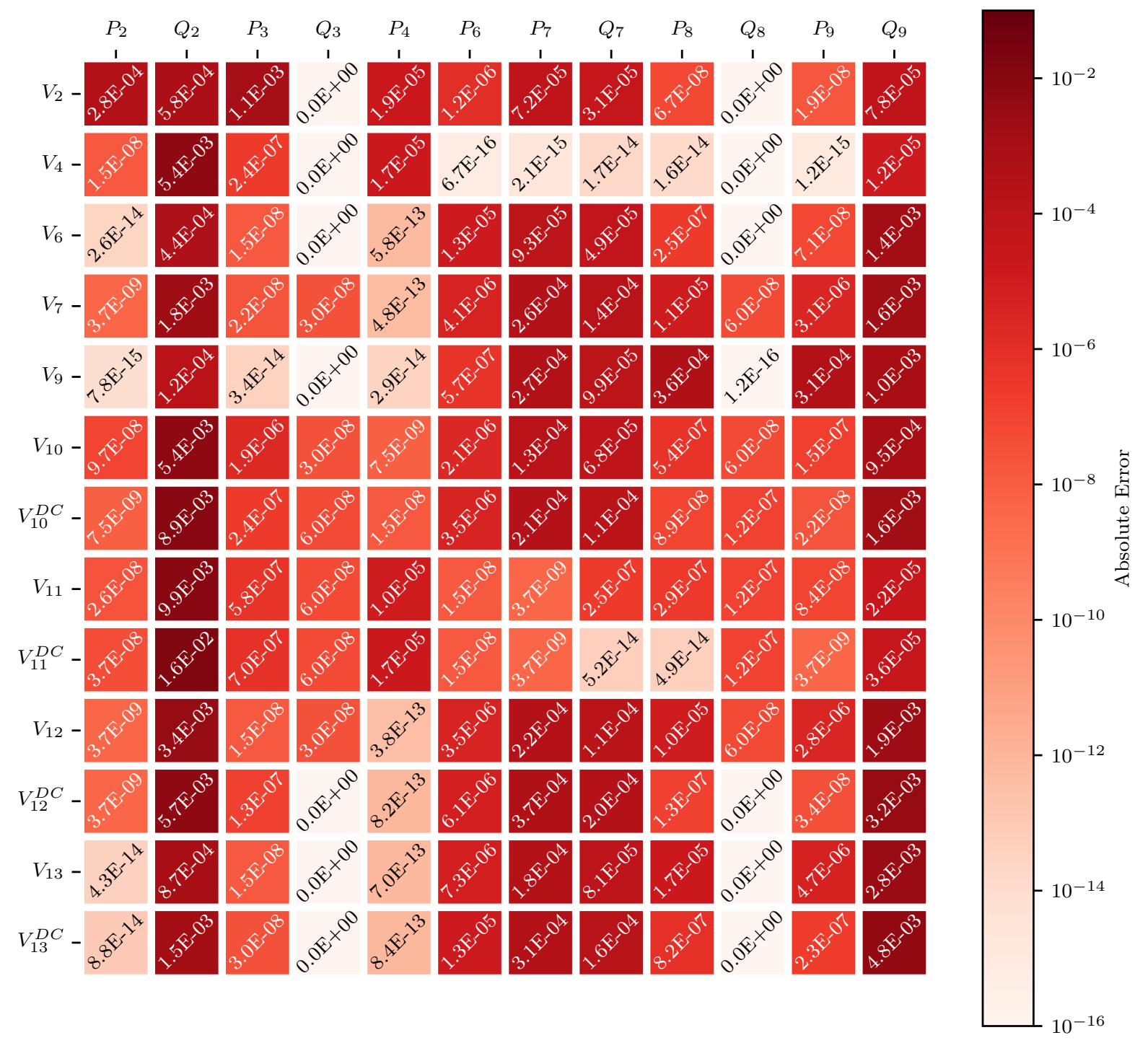

Figure 4. Absolute Error of Bus Voltage Magnitude Total Derivatives with Respect to Load Powers Computed with Analytic Derivatives and Finite Difference Approximations.

likely be required, a detailed analysis of the electrical distribution system was not completed as part of the design study. Therefore, this system was selected for analysis with the developed load flow tool as part of the present research. Ultimately, the intention is to couple the load flow electrical system model with other disciplinary models of this vehicle to develop a more detailed and realistic conceptual design.

As the initial conceptual design published by Johnson ${ }^{6}$ did not provide a detailed definition of the electrical distribution system layout, the first step was to define a potential layout for this system. The notional layout created as a baseline design for this study is shown in Figure 5. In this layout, a single turboshaft engine would be connected to the AC generator $(G)$ located at the bottom of the figure. This generator is assumed to provide power to bus 10 (shown as a horizontal bar) at 540 volts with a reference phase angle of zero. From bus 10 the current enters a rectifier $(R)$ converting it to DC current which is output to bus 9 for transmission throughout the aircraft. Transmission of power to the various loads on the system occurs on an interconnected set of of lines. The resistance of these lines were assumed to be 0.00096 ohms per meter in length, with line lengths approximated from the conceptual design drawings. A single DC load for auxiliary systems $\left(L_{A u x}\right)$ was included in the model and is shown at the center of the figure. This load is assumed to require a constant $2 \mathrm{~kW}$ of power. At the top of the figure, the DC transmission 
lines connect to four DC buses (buses 5 through 8) which each connect to an inverter $(I)$. The inverters are implemented such that the output voltage phase angle for the attached AC buses (buses 1 through 4 ) is zero. Attached to buses 1 through 4 are four AC loads $(L)$ which were each assumed to have a power factor of $95 \%$. A full description of the nominal design inputs for the tiltwing model is provided in Table 4 .

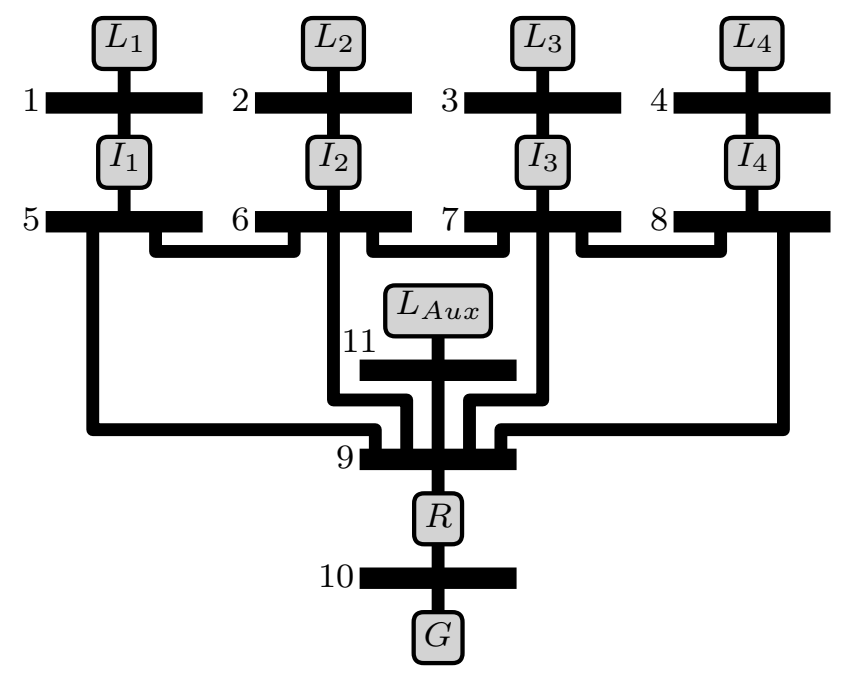

Figure 5. Tiltwing Electrical Distribution System Configuration.

Table 4. Nominal Tiltwing Electrical Distribution System Design Inputs.

\begin{tabular}{ccc|ccc}
\hline Design Input & Units & Value & Design Input & Units & Value \\
\hline$V_{10, m, i n}$ & $\mathrm{~V}$ & 540.0 & $P_{2}$ & $\mathrm{~W}$ & 565000.0 \\
$\theta_{10, i n}$ & $\mathrm{deg}$ & 0.0 & $Q_{2}$ & $\mathrm{~W}$ & 185706.5 \\
$\theta_{1-4, i n}$ & $\mathrm{deg}$ & 0.0 & $P_{3}$ & $\mathrm{~W}$ & 565000.0 \\
$R_{5,6}$ & $\Omega$ & 0.0029856 & $Q_{3}$ & $\mathrm{~W}$ & 185706.5 \\
$R_{6,9}$ & $\Omega$ & 0.0028224 & $P_{4}$ & $\mathrm{~W}$ & 565000.0 \\
$R_{7,8}$ & $\Omega$ & 0.0029856 & $Q_{4}$ & $\mathrm{~W}$ & 185706.5 \\
$R_{7,9}$ & $\Omega$ & 0.0028224 & $P_{\text {Aux }}$ & $\mathrm{W}$ & 2000.0 \\
$R_{5,9}$ & $\Omega$ & 0.00576 & $M_{\text {Rect }}$ & - & 0.99 \\
$R_{8,9}$ & $\Omega$ & 0.00576 & $\eta_{\text {Rect }}$ & - & 0.98 \\
$R_{6,7}$ & $\Omega$ & 0.00528 & $P F_{\text {Rect }}$ & - & 0.95 \\
$R_{9,11}$ & $\Omega$ & 0.0048 & $M_{\text {Inv }}$ & - & 0.99 \\
$P_{1}$ & $\mathrm{~W}$ & 565000.0 & $\eta_{\text {Inv }}$ & - & 0.98 \\
$Q_{1}$ & $\mathrm{~W}$ & 185706.5 & & & \\
\hline
\end{tabular}

While this electrical distribution system configuration may not ultimately be the setup for a real aircraft, the configuration provided a representative design that could be studied with the load flow analysis method. Therefore, using this notional model several different scenarios were evaluated to determine the potential design voltages, currents and power requirements. In addition, the analytic derivatives within the model were used to perform a sensitivity study. These scenarios and the resulting design implications are described in the following sections. Results for the scenarios in this section are shown graphically with tabular data included in Appendix A.

\section{Nominal Operation}

The first scenario considered the nominal hover operating condition for the tiltwing vehicle. In this scenario, a load of $565 \mathrm{~kW}$ was assumed for each of the four loads which represent the electric motors driving 
the propellers. With these loads, the load flow simulation was completed producing the results shown in Figure 6. In this figure, the diagram on the left depicts current through the DC transmission lines. The arrows on the lines indicate direction of the current flow with the color specifying the amperage. The results show the lines running from bus 9 to the interior inverter buses (6 and 7$)$ carry the most current. The majority of this current is used to power the interior motors with a small portion of the current continuing on to the outboard inverter buses (5 and 8) to power the outboard motors. However, most of the current for powering the outboard motors comes from the lines directly connecting buses 5 and 8 to bus 9 . Given the symmetry of the system and the loads, no current passes through the line connecting buses 6 and 7 .

The right diagram of the figure shows the voltage magnitude of each of the buses in the system. As mentioned in the beginning of this section, the generator provided power to bus 10 at a constant 540 volts. The remaining bus voltages decrease the farther away from the generator the bus is located, with the buses 1 and 4 having the lowest voltage magnitudes. Lastly, at the bottom of both the left and right diagrams, the figure shows that the generator is required to produce $2.3741 \mathrm{MW}$ of power to balance this system.
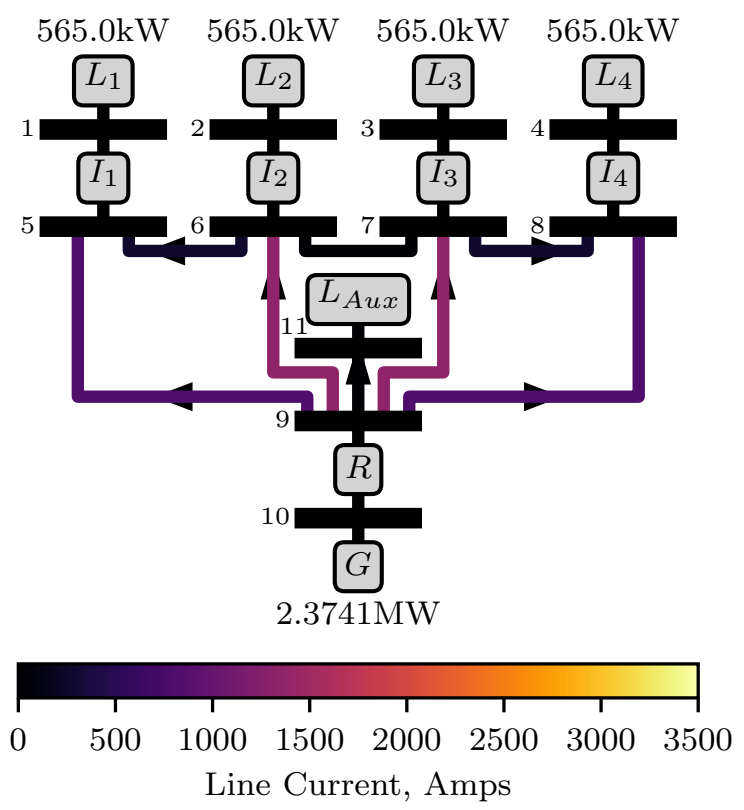
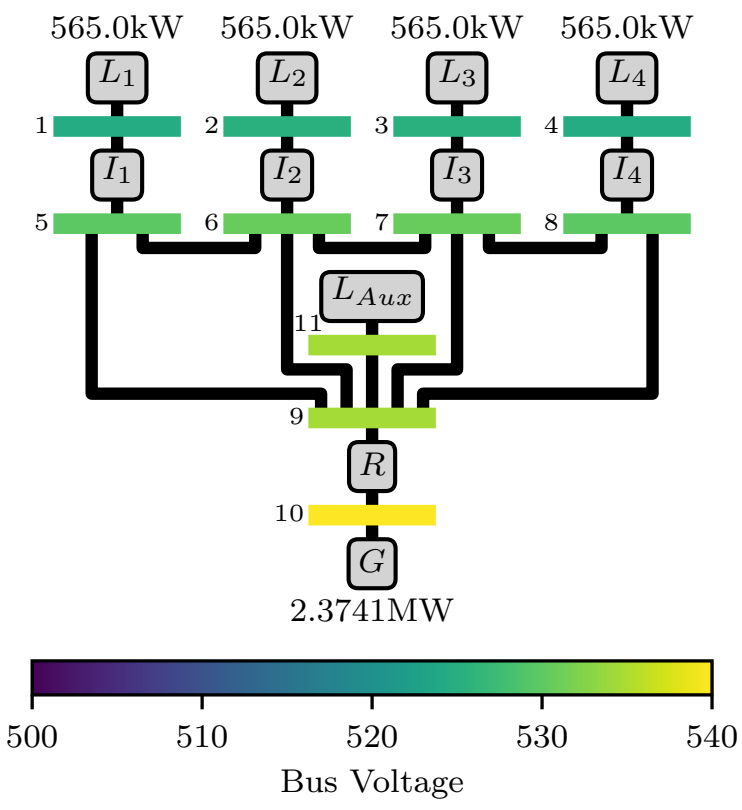

Figure 6. Tiltwing Electrical Distribution System Under Nominal Operation.

\section{Non-Uniform Load Distribution}

The second scenario examined a case were the power required by each of the loads varied across the span, possibly representing a turning maneuver. In this scenario, the power requirements on the left wing (loads 1 and 2) were decreased while the power requirements on the right wing (loads 3 and 4) were increased. However, the overall power requirement for all four loads was held equal to the nominal operating condition. The results from this scenario are shown in Figure 7 and are visually similar to those for nominal operation. However, given the higher power demands on the right wing, the lines going to the right wing carry slightly more current while those on the left wing carry less current. In addition, a small amount of current passes through the line connecting bus 6 to bus 7 . This line connects the left and right wings and was initially added to the model to provide redundancy in the event of faults in the transmission lines. However, this scenario shows that some current may pass through this line under normal operating conditions depending on the power demands in the system. 

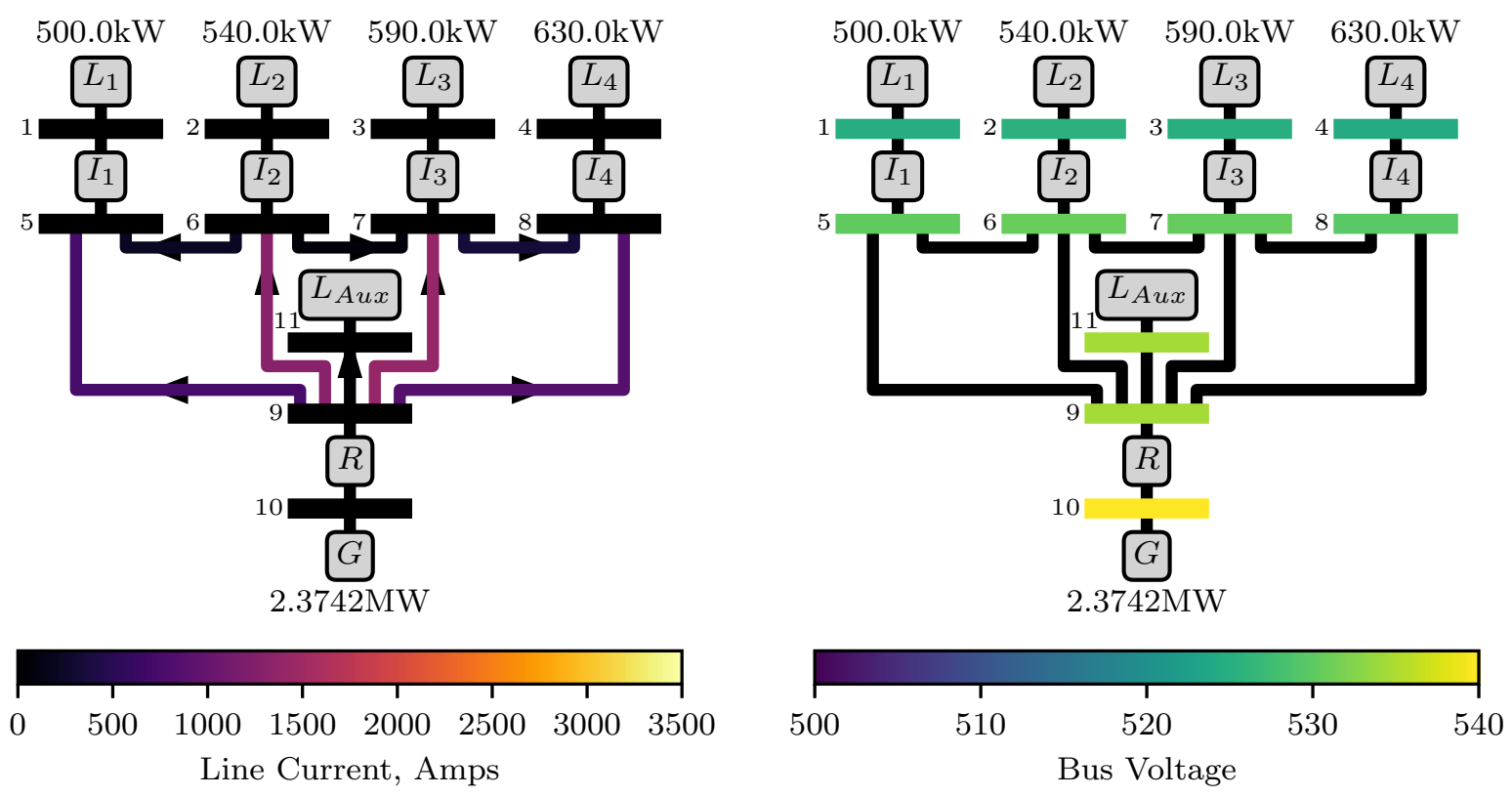

Figure 7. Tiltwing Electrical Distribution System with Non-Uniform Load Power Requirements

\section{Single Line Fault}

The third scenario examined the operation of the system when a fault occurred in the transmission line running from bus 9 to bus 6 . This fault was assumed to result in a complete loss of transmission capability through this line. Despite this fault, the scenario still specified that the same amount of power needed to be delivered to each of the loads as the nominal operating scenario. With this cut transmission line, the load flow simulation was able to find a solution as shown in Figure 8. In this figure, the cut transmission line is shown in gray with a red slash across it.

The results show that the current in the all remaining lines connected to bus 9 increase to transmit the required amount of power to the four loads, with the lines to both bus 5 and bus 7 increasing the most. Power is supplied to load 2 by sending some of this extra current through the lines connecting buses 5 and 7 to bus 6 . In this scenario, therefore, the direction of current flow on the line between inverters 1 and 2 reverse compared to the nominal operation scenario. Furthermore, the result shows the value of the redundancy line which connects the left and right wings (between buses 6 and 7 ) as this line helps redistribute current and power in the event of a fault. The diagram on the right also shows that the voltage on buses 2 and 5 decrease in order to achieve a steady operating condition. Lastly, the load flow analysis of this scenario determined that the overall power that needed to be produced by the generator increased slightly to 2.3875 MW. While the overall load power demands remained the same as the nominal operating scenario, the line fault required current take a longer path to load 2 resulting in increased losses in the lines.

\section{Double Line Fault}

The last scenario considered the operation of the electrical distribution system when faults occurred in two lines going to the same wing of the aircraft. These faults were again assumed to result in a complete loss of transmission capability for the lines between the bus 9 and buses 5 and 6 . As in the previous scenario, the overall power demands of the loads were held at the same values as the nominal operating case.

The load flow solution for this scenario is shown in Figure 9. The results in the left diagram show that the current on the line from bus 9 to bus 7 nearly triples from the nominal operating condition. The majority of this current then flows to the inverters and loads on the left wing via the lines running from bus 7 to bus 6 and bus 5 . The current on the line from the bus 9 to bus 8 also increases compared to the nominal scenario. The increased current on this line is used to support the power requirements of the rest of the system as the 

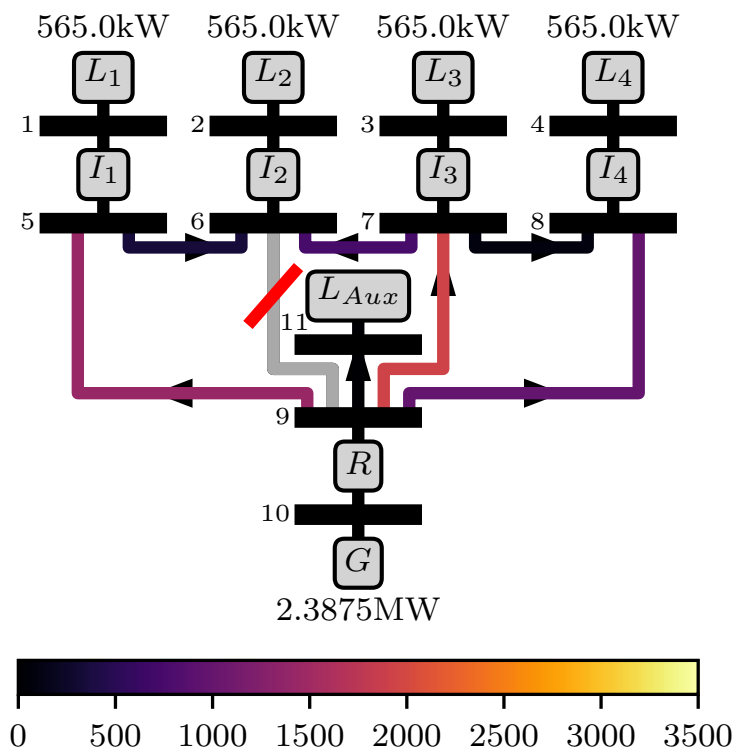

Line Current, Amps
$565.0 \mathrm{~kW} \quad 565.0 \mathrm{~kW} \quad 565.0 \mathrm{~kW} \quad 565.0 \mathrm{~kW}$

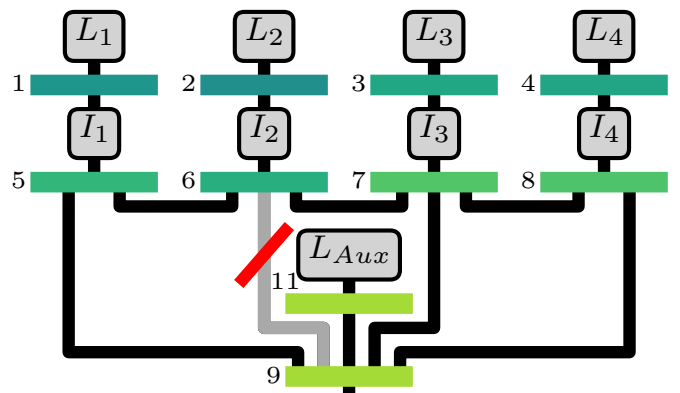

$R$

10

$G$

$2.3875 \mathrm{MW}$

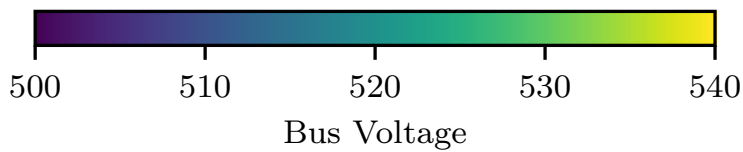

Figure 8. Tiltwing Electrical Distribution System with Single Line Loss

current in the line from bus 7 to bus 8 reverses direction compared to nominal operation. Achieving these current flows also necessitated a significant reduction in the bus voltages on the left wing compare to the previous scenario. Lastly, the double line fault in this scenario increased the power that must be produced by the generator by $2 \%$ to counteract the increased losses in longer transmission path.

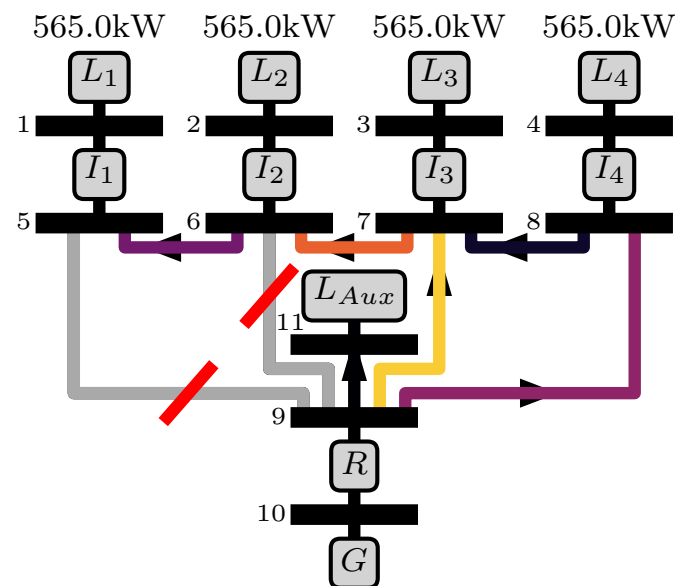

$2.4248 \mathrm{MW}$

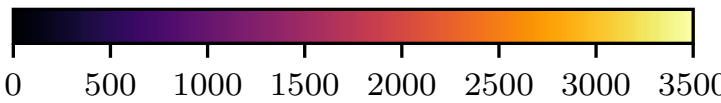

Line Current, Amps
$565.0 \mathrm{~kW} \quad 565.0 \mathrm{~kW} \quad 565.0 \mathrm{~kW} \quad 565.0 \mathrm{~kW}$

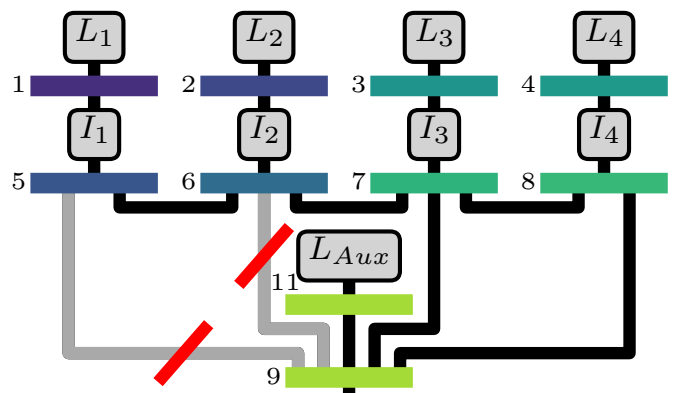

$R$

10

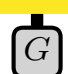

$2.4248 \mathrm{MW}$

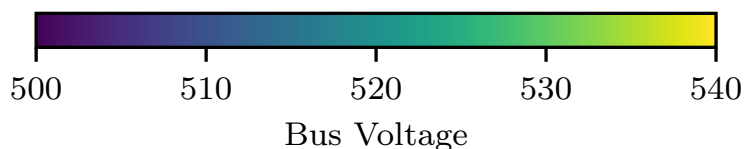

Figure 9. Tiltwing Electrical Distribution System with Double Line Loss

By examining this scenario in particular, the load flow analysis results show that the notional electrical system design provides a number of redundancies that can mitigate potential fault on multiple transmission 
lines. Furthermore, the results can provide useful information for determining the physical characteristics of the lines connecting the various buses such that they can provide satisfactory performance in potential fault scenarios. Lastly, examining scenarios such as this one can provide critical information for determining changes to turboshaft power requirements for emergency operation.

\section{Sensitivity Study}

In addition to evaluating the proposed tiltwing electrical distribution system design with the above scenarios, a simple sensitivity study was completed around the nominal operating condition. This study was used to provide insights about how changes to to a number of the design input values would impact the outputs of converged solution. This sensitivity study was completed using the analytic total derivatives computed by the developed analysis tool. The results in from the sensitivity study are given in Figure 10. The elements of the grid show the percent change in the output variables (listed on the left) from the nominal condition values for a one percent change in the design input value (listed across the top). The color of each of element indicates the strength of the sensitivity with blue colors being a positive correlation and red colors indicating a negative correlation.

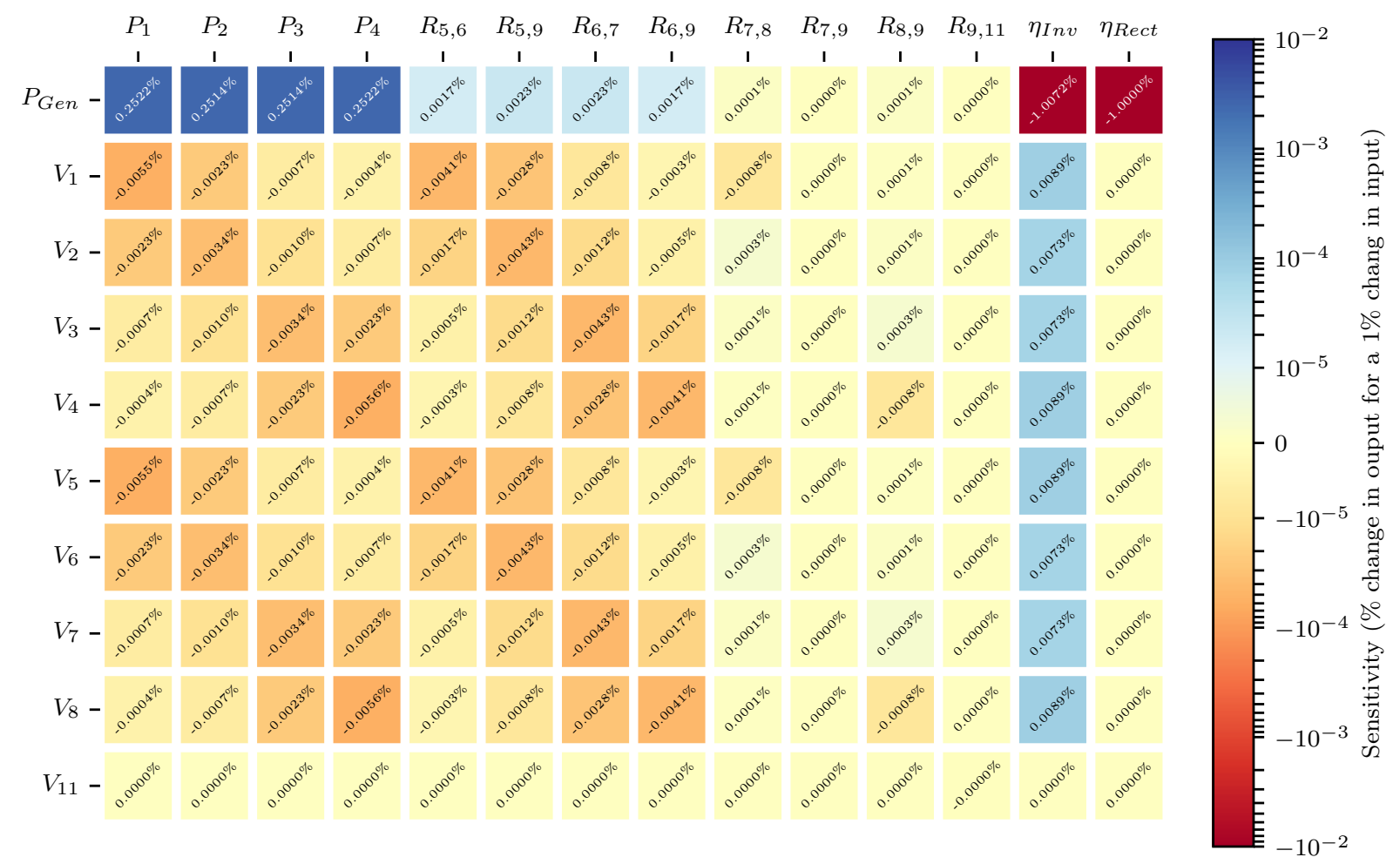

Figure 10. Sensitivity of Generator Power and Bus Voltages with Respect to Load Powers, Line Resistances and Converter Efficiencies.

In this figure, the first four columns give the sensitivity of the output generator power and bus voltages with respect to changes in the load powers. As would be expect, the required generator power has a strong positive correlation to the power values on each of the loads. While the numbers in the table are normalized for a $1 \%$ change to the nominal input value, the actual relationship is a $1 \mathrm{~W}$ increase in load power results in a $1.06 \mathrm{~W}$ increase in the required generator power. The generator power must increase more than the load power due to the losses in the lines. The first four columns also show that there is a slight negative correlation between changes in load powers and the bus voltage magnitudes. However, this correlation is not constant across all buses, as there are several darker orange clusters indicating stronger negative correlation. These darker clusters occur for the buses on the same wing of the vehicle as the load being changed (buses 
1, 2, 5, and 6 for loads 1 and 2 or buses $3,4,7$, and 8 for loads 3 and 4). This correlation is logical as an increase in a load on wing will necessitate a decrease in the bus voltages primarily on that side of the vehicle to draw more current for powering the load.

The next eight columns in the figure show the sensitivity of the generator power and voltages to changes in the individual line resistances. Examining these sensitivities is valuable for several reasons. First, the line resistances were based on an assumed line length and gauge. During the design process the length or gauge may change as the vehicle is sized to meet the mission requirements. Furthermore, the resistance of the lines will increase at higher line temperatures which are likely to result from power being lost in the lines. The results in the figure show a moderately positive correlation between increased line resistances and increased generator power demands. The results also show a slight negative correlation of the bus voltages for the increases in line resistances with the strongest correlations again being for those buses on the same wing as line resistance under consideration.

The last two columns show the sensitivity of the outputs with respect to the inverter and rectifier efficiencies. The efficiency of each of these components has a nearly one-to-one negative correlation with generator power. This result emphasizes the importance of the inverter and rectifier efficiencies on the overall viability of an electric aircraft propulsion system as each $1 \%$ drop in efficiency necessitates a $1 \%$ increase in generator power. The inverter efficiencies also have a moderate positive correlation with most of the bus voltages as this efficiency directly effects the power that must be delivered to each of the inverters. In contrast, the rectifier efficiency has no effect on the bus voltages throughout the system as the voltage on each side of the rectifier are set by the generator output voltage and rectifier modulation index.

\section{Conclusion and Future Work}

NASA and the aviation industry are researching a variety of new aircraft technologies that will make commercial aircraft more efficient by transitioning to alternative power and energy sources. Part of this research is focusing on electrified aircraft propulsion as it has the potential to reduce energy consumption, emissions and noise. A number of vehicle concepts have been proposed which this technology which span a broad range of aircraft sizes, configurations and missions. However, to date the conceptual level analysis of the electrical distribution systems for these vehicles have generally implemented simple models that do not provide extensive details regarding the electrical system design and performance. The research presented in this paper attempted to fill the gap by exploring the use of load flow analysis for the conceptual design of electrical distribution systems for these vehicle concepts.

The first part of this research focused on the development of a flexible and efficient load flow analysis capability within the OpenMDAO analysis framework. This framework was selected as it provides valuable features such as solvers and optimizers, and is being used to create a set of conceptual level analysis tools to enable multidisciplinary optimization of aircraft designs. Within the OpenMDAO framework, a set of 10 modular components were created which will enable the load flow analysis of most AC and DC electrical systems. These components included AC and DC lines, buses, generators and loads along with inverters and rectifiers. The creation of these components also included the analytical definition of the partial derivatives for each component to support the use of gradient based solvers and optimizers within OpenMDAO.

The next part of the research aimed to verify the created load flow analysis capability in OpenMDAO by reproducing the analysis of a published 13-bus hybrid AC-DC reference system. The individual load flow components were therefore combined to create a model of this system providing a test of each of the created components. The results from this analysis closely matched the published values (given some assumptions regarding line impedances) and verified the components were created correctly. In addition, the verification step compared the analytically computed derivatives with those determined through finite difference approximation. Both the partial and total analytic derivatives were found to closely match thereby validating the gradient calculations in the model.

The last part of the research effort explored the used of the verified load flow analysis capability to analyze the electrical distribution system for a turbo-electric tiltwing vehicle proposed for urban air mobility operations. The electrical distribution system for this concept vehicle was undefined, so a notional load flow model containing both AC and DC subsystems was proposed for this study. The model was then used to examine several different operating scenarios which varied load power requirements as well as introduced faults into the system. Analysis results from these scenarios provided information about the voltages and currents throughout the system. Furthermore, the fault scenarios showed the importance of adding redundant 
lines to the system as those lines enabled full power to be delivered to the loads even in the case of two faults. These fault scenarios also provided an indication of the changes to the line currents and required generator power which must be considered for these situations as part of the design process.

Overall, this research found that load flow analysis can be used within the conceptual design process for electrified aircraft to provide a more thorough analysis of the electrical system design. The developed load flow analysis capability in OpenMDAO was also found to be flexible and efficient enabling the rapid design, analysis and optimization of these concepts. These features will be valuable as this tool will be used in future research efforts into electric aircraft conceptual design. These research efforts will likely include further verification studies and application of the developed tool to electric distribution systems for other vehicle concepts. Lastly, the developed load flow analysis tool will be applied as part of a multidisciplinary optimization process for electric UAM aircraft concepts. This multidisciplinary analysis will also consider analysis of the gas turbine engine, propeller design, thermal management system, aircraft structures, and mission performance. The electrical system has direct ties to most of these disciplines making the integrated design of these systems with tools such as load flow analysis critical to generating viable vehicle designs.

\section{Acknowledgments}

The work presented in this paper was supported by NASA's Transformative Tools and Technologies (TTT) Project. The authors would like to thank Justin Gray, Raymond Beach, Ralph Jansen, Timothy Dever, and Peter Kascak of NASA GRC for their guidance in this research. Lastly, the authors would like to thank the OpenMDAO development team for their technical support of this effort.

\section{References}

\footnotetext{
1 "NASA Aeronautics Strategic Implementation Plan: 2017 Update," 2017, NP-2017-01-2352-HQ.

${ }^{2}$ Jansen, R. H., Bowman, C., Jankovsky, A., Dyson, R., and Felder5, J., "Overview of NASA Electrified Aircraft Propulsion Research for Large Subsonic Transports," 53rd AIAA/SAE/ASEE Joint Propulsion Conference, AIAA, Atlanta, GA, July 2017, AIAA 2017-4701.

${ }^{3}$ Borer, N. K., Patterson, M. D., Viken, J. K., Moore, M. D., Bevirt, J., Stoll, A. M., and Gibson, A. R., "Design and Performance of the NASA SCEPTOR Distributed Electric Propulsion Flight Demonstrator," 16th AIAA Aviation Technology, Integration, and Operations Conference, Washington, D.C., 2016, AIAA-2016-3920.

${ }^{4}$ Welstead, J. R. and Felder, J. L., "Conceptual Design of a Single-Aisle Turboelectric Commercial Transport with Fuselage Boundary Layer Ingestion," 54th AIAA Aerospace Sciences Meeting, San Diego, CA, 2016, AIAA-2016-1027.

${ }^{5}$ Felder, J. L., Brown, G. V., Kim, H. D., and Chu, J., "Turboelectric Distributed Propulsion in a Hybrid Wing Body Aircraft," 20th International Society for Airbreathing Engines, Gothenburg, Sweden, 2011, ISABE-2011-1340.

${ }^{6}$ Johnson, W., Silva, C., and Solis, E., "Concept Vehicles for VTOL Air Taxi Operations," AHS International Technical Meeting on Aeromechanics Design for Transformative Vertical Flight, San Francisco, CA, 2018.

${ }^{7}$ Bradley, M. K., Allen, T. J., and Droney, C., "Subsonic Ultra Green Aircraft Research: Phase II-Volume III-Truss Braced Wing Aeroelastic Test Report," Tech. rep., NASA, April 2015, NASA-CR-2015-218704.

${ }^{8}$ Schiltgen, B. T. and Freeman, J., "Aeropropulsive Interaction and Thermal System Integration within the ECO-150: A Turboelectric Distributed Propulsion Airliner with Conventional Electric Machines," 16th AIAA Aviation Technology, Integration, and Operations Conference, Washington, D.C., 2016, pp. AIAA-2016-4064.

${ }^{9}$ Falck, R. D., Chin, J., Schnulo, S. L., Burt, J. M., and Gray, J. S., "Trajectory Optimization of Electric Aircraft Subject to Subsystem Thermal Constraints," 18th AIAA/ISSMO Multidisciplinary Analysis and Optimization Conference, Denver, CO, 2017, AIAA-2017-4002.

${ }^{10}$ Glover, J. D. and Sarma, M., Power System Analysis and Design, PWS-KENT, Boston, 1987.

${ }^{11}$ Ahmed, H. M., Eltantawy, A. B., and Salama, M., "A Generalized Approach to the Load Flow Analysis of AC-DC Hybrid Distribution Systems," IEEE Transactions on Power Systems, Vol. 33, No. 2, 2017, pp. 2117-2127.

${ }^{12}$ Chapman, J. W. and Litt, J. S., "An Approach for Utilizing Power Flow Modeling for Simulations of Hybrid Electric Propulsion Systems," AIAA Electric Aircraft Technologies Symposium, AIAA, Cincinnati, OH, July 2018, AIAA 2018-5018.

${ }^{13}$ OpenMDAO, www.openmdao.org, Accessed: 2018-06-05.

${ }^{14}$ Gray, J. S., Hearn, T. A., Moore, K. T., Hwang, J., Martins, J., and Ning, A., "Automatic Evaluation of Multidisciplinary Derivatives Using a Graph-Based Problem Formulation in OpenMDAO," 15th AIAA/ISSMO Multidisciplinary Analysis and Optimization Conference, 2014, AIAA-2014-2042.

${ }^{15}$ Martins, J. R. R. A. and Hwang, J. T., "Review and Unification of Methods for Computing Derivatives of Multidisciplinary Computational Models," AIAA Journal, Vol. 51, No. 11, November 2013, pp. 2582-2599.

${ }^{16}$ Hwang, J. T., A Modular Approach to Large-Scale Design Optimization of Aerospace Systems, PhD dissertation, University of Michigan, School of Aerospace Engineering, 2015.
} 


\section{A. Notional Turbo-Electric Tiltwing Electric Distribution System Results}

The tables in this appendix provide details of the turbo-electric tiltwing scenario results presented in Section IV. Table 5 provides the currents through the DC lines. In this table, positive current values indicate flow from the bus in the first column to the bus in the second column with negative numbers indicating flow in the opposite direction. Table 6 provides the voltage magnitudes for both the AC and DC buses in the system. All voltage phase angles were set to zero and are therefore not included in the table.

Table 5. Notional Turbo-Electric Tiltwing Electric Distribution System DC Currents, Amps.

\begin{tabular}{cccccc}
\hline From Bus & To Bus & Nominal Operation & Non-Uniform Load & Single Line Fault & Double Line Fault \\
\hline 9 & 5 & 811.2556 & 750.6135 & 1447.3139 & 0.0000 \\
9 & 6 & 1362.9373 & 1308.0991 & 0.0000 & 0.0000 \\
9 & 7 & 1362.9373 & 1417.8797 & 1930.4291 & 3075.2226 \\
9 & 8 & 811.2556 & 871.9881 & 995.1417 & 1366.1017 \\
9 & 11 & 3.7412 & 3.7412 & 3.7412 & 3.7412 \\
6 & 5 & 276.6875 & 211.5336 & -351.7969 & 1128.9758 \\
7 & 8 & 276.6875 & 341.9171 & 94.9803 & -271.5576 \\
6 & 7 & 0.0000 & 58.6827 & -745.9110 & -2250.5487 \\
\hline
\end{tabular}

Table 6. Notional Turbo-Electric Tiltwing Electric Distribution System Voltage Magnitudes and Phase Angles

\begin{tabular}{ccccc}
\hline Bus & Nominal Operation & Non-Uniform Load & Single Line Fault & Double Line Fault \\
\hline 1 & 524.6279 & 524.9737 & 521.0008 & 505.5603 \\
2 & 525.4457 & 525.5989 & 519.9610 & 508.8972 \\
3 & 525.4457 & 525.2922 & 523.8600 & 520.6613 \\
4 & 524.6279 & 524.2816 & 523.5793 & 521.4639 \\
5 & 529.9272 & 530.2765 & 526.2635 & 510.6669 \\
6 & 530.7532 & 530.9080 & 525.2131 & 514.0376 \\
7 & 530.7532 & 530.5982 & 529.1516 & 525.9205 \\
8 & 529.9272 & 529.5773 & 528.8680 & 526.7313 \\
9 & 534.6000 & 534.6000 & 534.6000 & 534.6000 \\
10 & 540.0000 & 540.0000 & 540.0000 & 540.0000 \\
11 & 534.5820 & 534.5820 & 534.5820 & 534.5820 \\
\hline
\end{tabular}

